GOLD stage II COPD patients had reduced lung function, quadriceps strength and $6 \mathrm{MW}$ test despite a similar smoking history. There was increased mitochondrial and intracellular ROS in both skeletal muscle and bronchial biopsies of COPD patients compared to controls. There was a trend for reduced MP in COPD EB mitochondria.

Conclusion The presence of excessive ROS in cells from a lung and a non-lung compartment support the existence of a generalised dysfunction of mitochondria in established COPD resulting in increased mitochondrial oxidative stress.

\section{P190 CONTRASTING TECHNIQUES FOR THE STUDY OF COPD LUNG MICRO-STRUCTURE WITH X-RAY MICRO- COMPUTED TOMOGRAPHY}

RS Smith, AS Scott, IS Sinclair, JW Warner, PL Lackie; Southampton University, Southampton, England

\subsection{6/thoraxjn-2013-204457.342}

Background Chronic obstructive pulmonary disease (COPD) is a severely debilitating lung condition characterised by airway obstruction within the distal respiratory tree. Micro-CT imaging is a novel radiographic method that can generate a three-dimensional reconstruction of human lung micro-structure at resolutions approaching $1 \mu \mathrm{m}$. This has revealed an obliteration of terminal bronchioles that may begin before patients have symptoms. A significant challenge when imaging wax-embedded COPD lung tissue is improving the contrast-to-noise for reasonable scan durations. The low contrast within the tissue can result in image analysis taking weeks to perform so addressing this

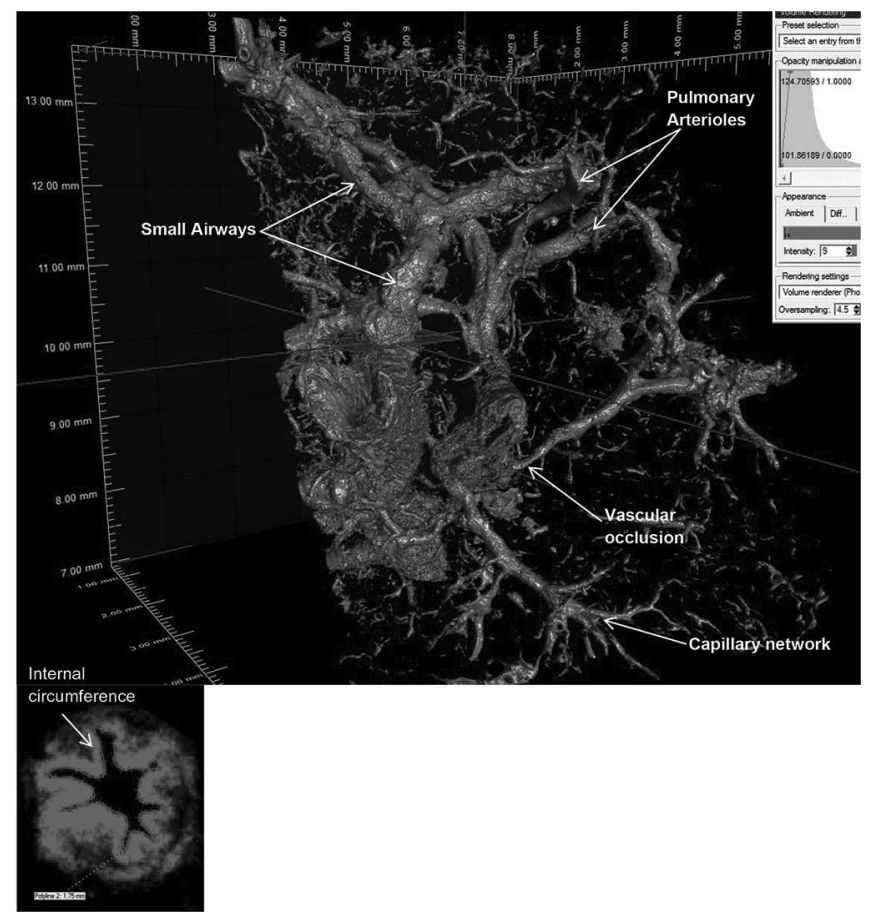

Abstract P190 Figure 1. After segmentation. Small airways and accompanying vessels were almost entirely segmented after $0.1 \%$ PTA staining by quickly increasing the threshold applied to the subvolume (see the higher threshold values displayed on the histogram). The blue marker shows where the airway cross section was taken (bottom left). Measuring internal circumference proved to be highly precise. issue is critical if micro-structure is to be studied in a more robust and less time-consuming fashion.

Aims The study's aim was to compare and quantify the effects of different contrasting techniques on the ability of micro-CT to visualise small airways $<2 \mathrm{~mm}$ in diameter and micro-vasculature in COPD human lung tissue.

Methods Samples were obtained from formalin-fixed sub-pleural lung tissue resected from a patient with moderate COPD and were incubated in $0.1 \%$ phosphotungstic acid (PTA), 25\% Lugol's iodine, $1 \%$ silver nitrate or left unstained to act as a control. Post-incubation, samples were embedded in epoxy resin or paraffin wax and then imaged with a $225 \mathrm{kV}$ HMX CT scanner at Southampton University with an average voxel size of $7.6 \mu \mathrm{m}$ The data was then analysed in Image J and VGI StudioMax.

Results Staining with $0.1 \%$ PTA and 25\% Lugol's iodine significantly improved $\mathrm{x}$-ray contrast $(\mathrm{p}<0.01)$ with most intense staining occurring in the small airways and micro-vessels. Staining with $1 \%$ silver nitrate failed to improve contrast $(\mathrm{p}=0.110)$. PTA staining enabled small airway and vascular occlusions to be three-dimensionally characterised, providing reliable quantification of the micro-structure. Fast and simple image segmentation taking 10 minutes was required to effectively map out most of the branching network of small airways and micro-vessels. Visualising micro-structure in uncontrasted control samples required complex image analysis which took four hours to complete.

Conclusion PTA staining is a simple and effective technique at increasing $\mathrm{x}$-ray contrast and reducing noise in COPD lung tissue. This greatly improves the level of visualisation of microstructure in COPD tissue, providing more efficient and reliable analysis.

\section{P191 BETA-2 ADRENOCEPTOR GENOTYPE AND RESPONSE TO PROPRANOLOL IN PATIENTS WITH PERSISTENT ASTHMA}

WJ Anderson, PM Short, JLR Lipworth, A Manoharan, CNA Palmer, BJ Lipworth; University of Dundee, Dundee, Scotland, UK

\subsection{6/thoraxjnl-2013-204457.343}

Background The arginine-16-glycine (Arg16Gly) beta-2 adrenoceptor (ADR) polymorphism is associated with worse outcomes in patients exposed to regular beta-agonists. We therefore wished to know if Arg16Gly conferred a similar effect in response to beta-antagonists in asthma.

Methods and observations We have performed a retrospective composite analysis of two randomised controlled trials looking at effects of Arg16Gly on the chronic response to propranolol in $\mathrm{n}=25$ mild to moderate corticosteroid treated persistent asthmatics. We evaluated chronic dosing effects of propranolol given for at least 4 weeks ( $80 \mathrm{mg}$ dose at least 2 weeks) on pulmonary

\begin{tabular}{|c|c|c|c|c|c|}
\hline & \multicolumn{2}{|c|}{ ArgArg or ArgGly } & \multicolumn{2}{|l|}{ GlyGly } & \multirow{3}{*}{$\begin{array}{l}\text { Genotype } \\
\text { Comparison } \\
\text { P-value }\end{array}$} \\
\hline & $n=15$ & & $n=10$ & & \\
\hline & Change from & P-value & Change from & P-value & \\
\hline & Baseline (SEM) & & Baseline (SEM) & & \\
\hline FEV1 (\%) & $-3.9(1.2)$ & 0.006 & $-2.3(3.4)$ & 0.51 & 0.66 \\
\hline FEF25-75 (\%) & $-5.2(2.1)$ & 0.025 & $+2.0(3.2)$ & 0.55 & 0.06 \\
\hline R5 (\%) & $+20.7(5.5)$ & 0.003 & $+6.0(10.3)$ & 0.57 & 0.27 \\
\hline Recovery FEV1 (\%) & $-14.3(3.0)$ & $<0.001$ & $-8.4(2.8)$ & 0.014 & 0.18 \\
\hline
\end{tabular}

